$16^{\text {th }}$ International Conference on

AEROSPACE SCIENCES \& AVIATION TECHNOLOGY,

$\boldsymbol{A S A T}$ - 16 - May 26 - 28, 2015, E-Mail: asat@mtc.edu.eg

Military Technical College, Kobry Elkobbah, Cairo, Egypt

Tel : +(202) 24025292 - 24036138, Fax: +(202) 22621908

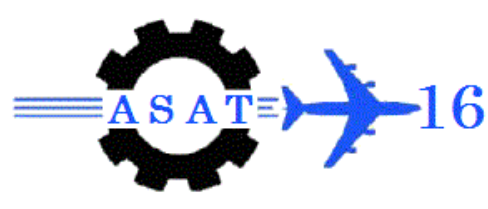

\title{
Characterization of MagnetoRheological Dampers using Existing Parametric Models
}

\author{
H.A.Hammad*, I. Saleh Mostafa*, I. A. Elsherif*, A. M. Salem*
}

\begin{abstract}
Magnetorheological (MR) dampers have received great deal of attention in recent years as they are one of the most promising controlled devices.

Due to the inherent nonlinear nature of magnetorheological (MR) dampers, one of the challenging aspects for developing and utilizing these devices to achieve high performance is the development of models that can accurately describe their unique characteristics. In this paper, different kinds of existing parametric models are discussed where their parameters have been studied. The experimental data including displacement, velocity and force are measured for different values of current. These models are verified by experimental measurements to obtain the more accurate model.
\end{abstract}

Keywords: semi active suspension system, smart fluid, magnetorheological fluid.

\section{Introduction}

Magnetorheological fluids were first discovered by the inventor Willis Winslow, who achieved a US patent regarding these fluids in 1947 [1] and published a scientific article in 1949 [2]. The yield stress of these fluids can be controlled very precisely by changing the field intensity to generate a continuously variable damping force. An MR fluid consists of micro-sized magnetically polarisable particles, mainly iron particles, suspended in a carrier liquid like mineral oil, synthetic oil, water or glycol. A typical MR fluid contains 20 to 40 percent by volume of relatively pure iron particles around 3 to 10micron diameter in size [3]. A variety of proprietary additives, similar to those found in commercial lubricants, is commonly added.

These additives are intended to discourage gravitational settling and promote particle suspension, enhance lubricity, modify the viscosity and inhibit wear. MR fluids respond to a magnetic field with a dramatic change in rheological behavior.

\footnotetext{
* Egyptian Armed Forces
} 
Moreover, MR fluids can be reversibly and instantaneously changed from a free-flowing liquid to a semi-solid within a few milliseconds with controllable yield strength when subjected to a magnetic field [3]. In the absence of an applied field, an MR fluid is reasonably well approximated as a Newtonian liquid; it is free flowing with a consistency similar to motor oil. In this condition, the ferrous particles are in an amorphous state as shown in figure 1(a). When a magnetic field is applied, the ferrous particles begin to align along the flux path, as shown in figure 1(b), eventually forming particle chains in the fluid, as shown in figure 1(c). Such chains resist and restrict fluid movement. As a result, a yield stress is developed in the fluid. The degree of change is related to the strength of the applied magnetic field and it has been shown that this change can occur in less than 1 millisecond [4].

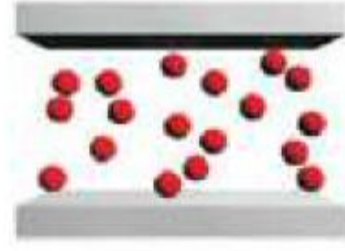

(a)

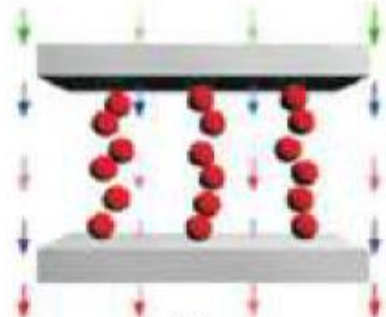

(b)

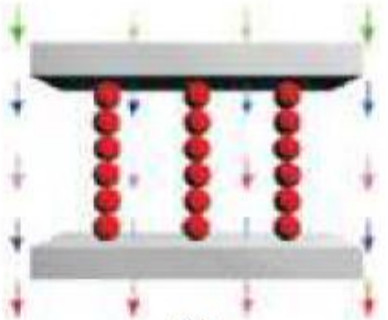

(c)

Fig.1. Activation of MR fluids [6], (1-a) No magnetic field applied, (1-b) partially magnetic field applied, (1-c) full magnetic field applied.

The controllable yield stress is exploited by MR fluid devices, whose mode of operation is classified according to the way their MR fluid is made to flow. These devices are classified as operating in one of three basic modes, or a combination of these modes: (a) valve mode (b) direct shear mode (c) squeeze mode.

Diagrams of these basic modes of operation are shown in figure (2). Examples of valve mode devices include servo-valves, dampers and actuators. Shear mode devices include clutches, brakes, dampers and structural composites. While less well-understood than the other modes, the squeeze mode has been used in some small-amplitude vibration dampers [5].

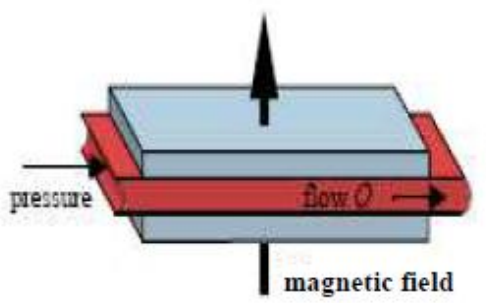

(a)

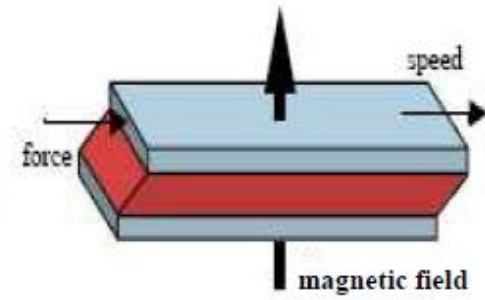

(b)

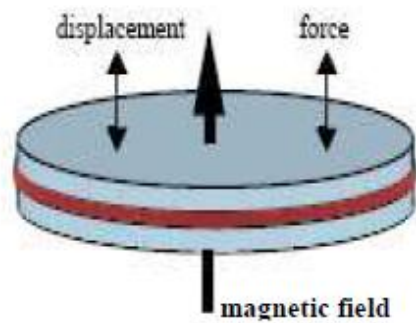

(c)

Fig. 2. Basic operating modes for controllable fluid devices [7], (2-a) valve mode, (2-b) direct shear mode, (2-c) squeeze mode.

\section{Experimental test setup}

The prototype MR damper obtained for evaluation is a fixed orifice damper filled with a magnetorheological fluid developed by the Lord Corporation as shown in figure (3). 


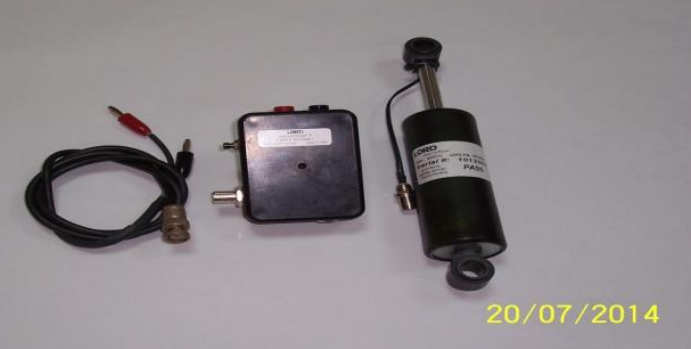

Fig. 3. MR fluid damper with control box and cables.

The damper is $24 \mathrm{~cm}$ long in its extended position, and the main cylinder is $3.8 \mathrm{~cm}$ in diameter. The main cylinder houses the piston, the magnetic circuit, an accumulator and $50 \mathrm{ml}$ of MR fluid, and the damper has a $4.4 \mathrm{~cm}$ stroke. As shown in figure (4).

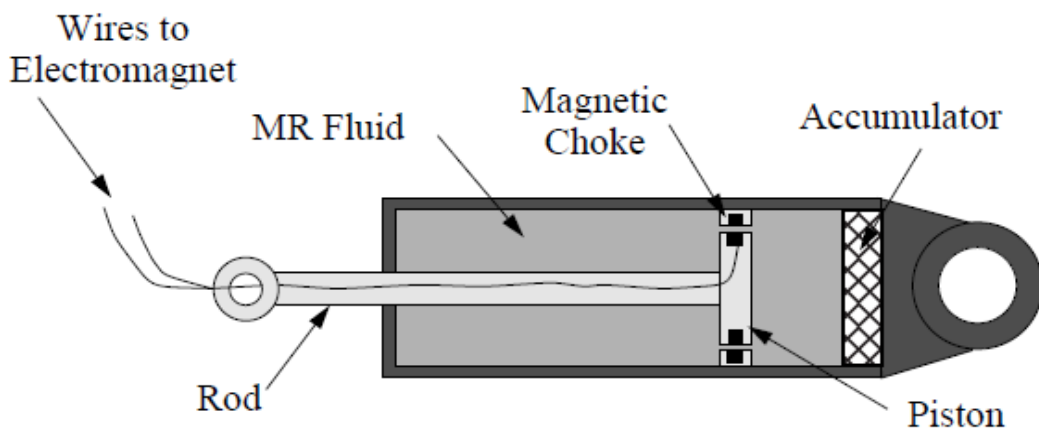

Fig. 4. Schematic of MRF damper.

The MR fluid valve is contained within the damper piston and consists of an annular flow channel having an inner diameter of $27 \mathrm{~mm}$ and an outer diameter of $28 \mathrm{~mm} \mathrm{[8].} \mathrm{The}$ magnetic field is applied radially across the resulting $0.5 \mathrm{~mm}$ dimension, perpendicular to the direction of fluid flow. The total axial length of the flow channel is $15 \mathrm{~mm}$ of which $7 \mathrm{~mm}$ is exposed to the applied magnetic field.

The damper test system (MTS 793) used for measuring the dynamic characteristics of magnetorheological fluid (MRF) damper is shown in Figure (5). The system consists of, the loading frame, the control unit and the hydraulic power unit (HPU). The loading unit has two vertical columns with adjustable upper crosshead and base (fixed to the frame). On the upper crosshead frame there is a fixture to clamp the upper end of the damper. Where the load cell is attached for measuring the generated damping force. The actuator mounted on the lower frame has a built-in linear voltage displacement transducer (LVDT) to measure the displacement of the damper moving end. 


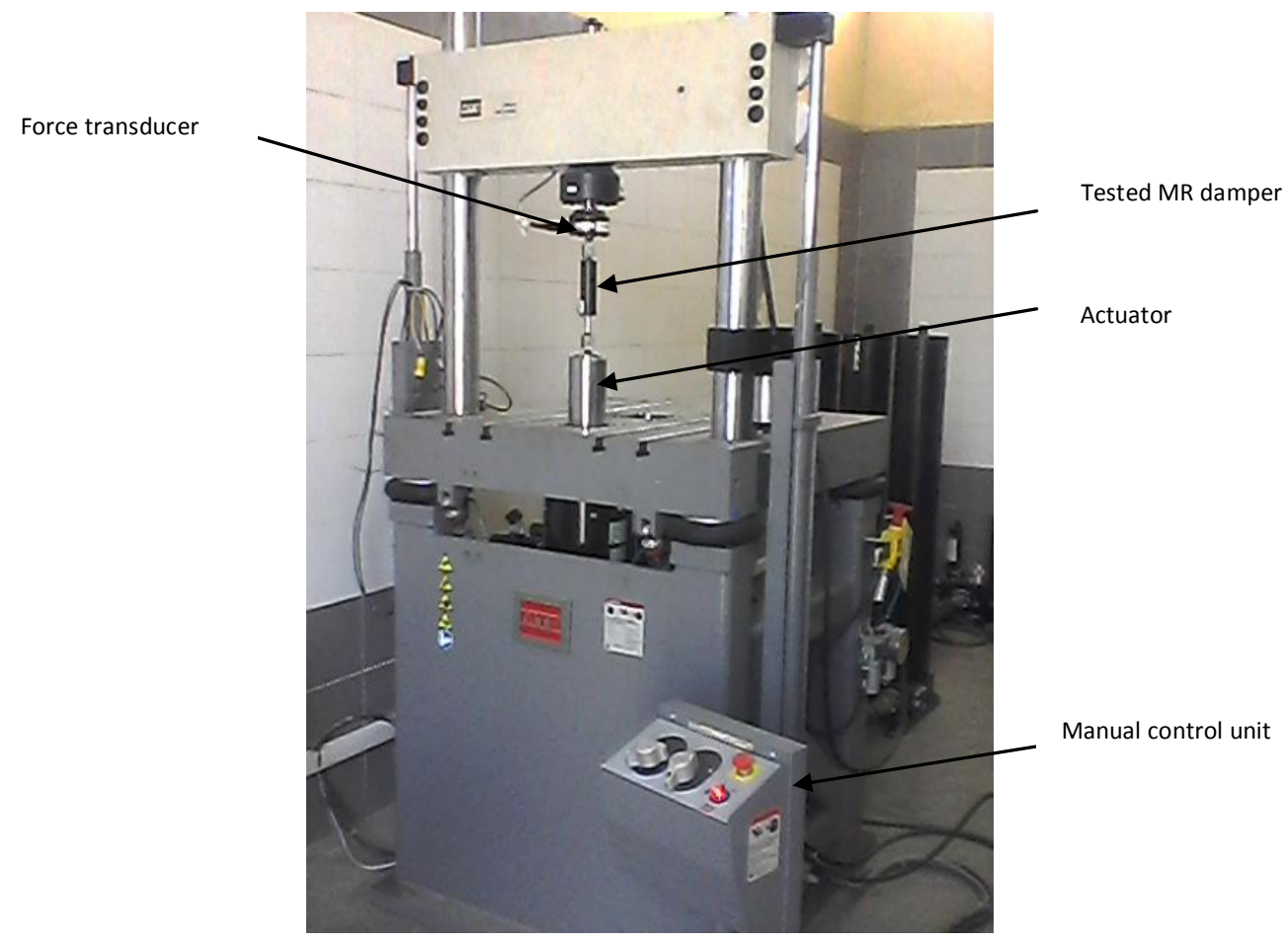

Fig. 5. Damper test system (793) used to identify MR damper characteristics.

The lower crosshead frame has a clamp to fix the lower end of the damper. The control unit is used to adjust the frequencies and amplitudes of the excitation. The unit has two channels to measure the displacement and force signals which can be directed to a computer through a data acquisition card. In the case of the MR damper test, the current driver needed for activating the MR fluid is provided by a $12 \mathrm{~V}$ DC power supply.

The stroke of the damper was measured before installation to the machine and a midpoint which equals to half of the stroke is marked on the piston rod. This will help to adjust the piston to its mid point to prevent the damper from damage. The damper was excited by sinusoidal excitations at a wide range of frequencies and amplitudes. This range represents the loading operational conditions of the dampers. The tests are carried out with peak to peak amplitudes of excitations ranging from $5 \mathrm{~mm}$ to $40 \mathrm{~mm}$. Choosing $40 \mathrm{~mm}$ as a maximum amplitude value since the maximum stroke of the actuator rod is restricted to $( \pm 20 \mathrm{~mm})$. For the MR damper active state test, the damper is tested when the coil is energized with a range of current from $(0.25-1)$ Amp.

Different combinations of frequencies of 1,2, 3 and $5 \mathrm{~Hz}$ and amplitudes of 5, 10, 15 and 20 $\mathrm{mm}$ are used during measurements. The same combinations are repeated at $0.25,0.5,0.75$ and 1 Amp during testing of the MR damper in its active state.

Figure $(6-a, b, c)$ shows the force - time, force - displacement, and force - velocity respectively. 


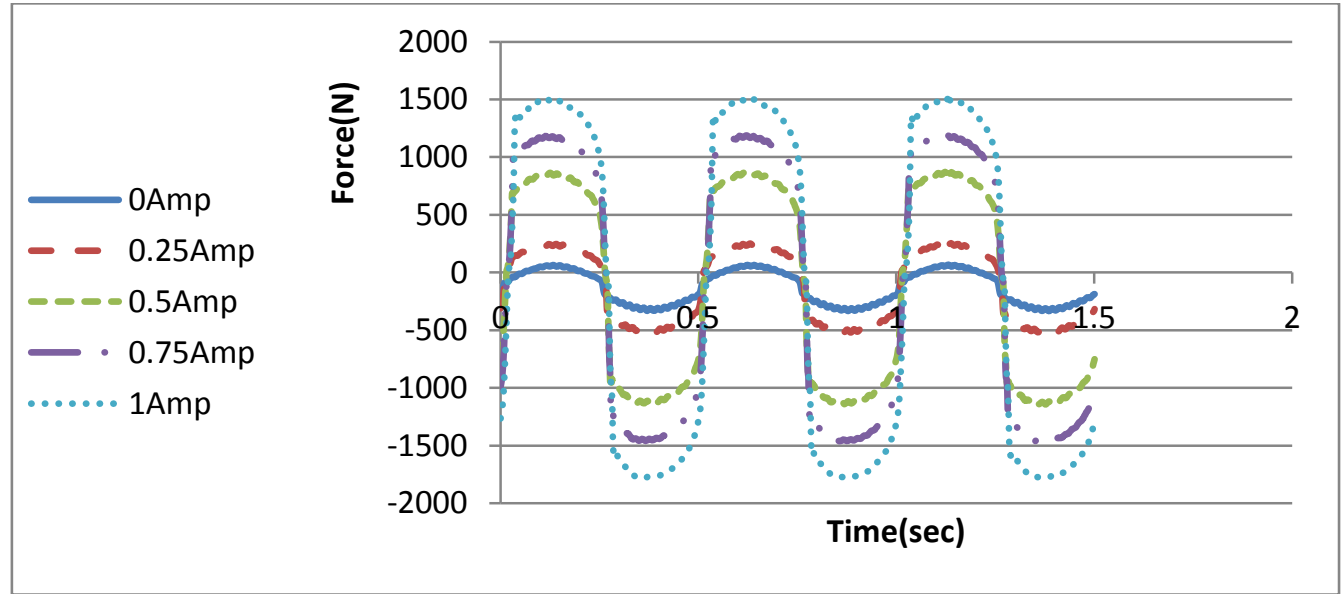

(a)
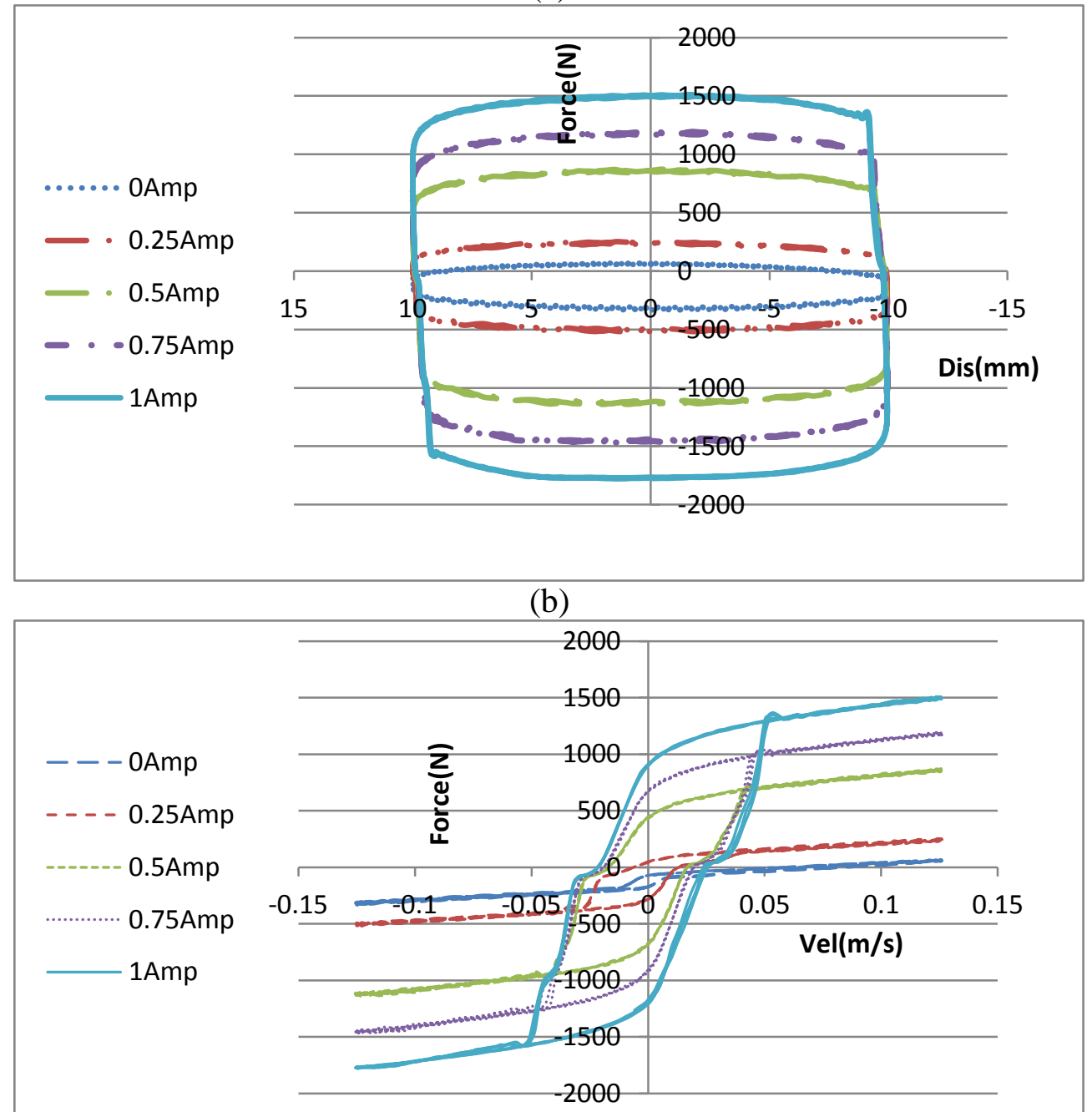

(c)

Fig. 6. Experimentally measured characteristics of MR damper for $2 \mathrm{~Hz}$ sinusoidal excitation with amplitude $20 \mathrm{~mm}$ and different currents.

In figure (6), the effect of changing the magnetic field is readily observed. At 0 current, the MR damper primarily exhibits the characteristics of a purely viscous device (i.e., the forcedisplacement relationship is approximately elliptical, and the force-velocity relationship is nearly linear).

However, as the current increases, the force required to yield the MR fluid in the damper increases and produces behavior associated with a plastic material in parallel with a viscous 
damper, i.e., Bingham plastic behavior (Shames and Cozzarelli 1992). Also, notice that the increase in force for a given increase in the applied current is approximately linear for current between 0-1 Amperes.

The deviation of the force produced by the damper from zero position as seen from figure (6a) is due to the presence of an accumulator in the MR damper. This consists of a bladder within the main cylinder that is filled with nitrogen pressurized at 300 psi. The accumulator helps prevent cavitations in the fluid during normal operation and accounts for the volume of fluid displaced by the piston rod as well as thermal expansion of the fluid.

\section{Dynamic model for MR damper.}

The stress-strain behavior of the Bingham viscoplastic model (Shames and Cozzarelli, 1992) is often used to describe the behavior of MR fluids. In this model, the plastic viscosity is defined as the slope of the measured shear stress versus shear strain rate data. Thus, for positive values of the shear rate $(\gamma)$, the total stress is given by $[8,9,10]$ :

$\tau=\tau_{y(\text { field })}+\eta \dot{\gamma}$

Where $\tau_{y(f i e l d)}$ is the yield stress induced by the magnetic field and $\eta$ is the viscosity of the fluid.

Based on this model of the rheological behavior of MR fluids, Stanway, et al. $(1985,1987)$ proposed an idealized mechanical model, denoted the Bingham model, for the behavior of an MR damper. The Bingham model consists of a Coulomb friction element placed in parallel with a viscous damper, as shown in figure (7). In this model, for nonzero piston velocities $\left(x^{\circ}\right)$, the force generated by the device given by:

$F=f_{c} \operatorname{sgn}(\dot{x})+c_{o} \dot{x}+f_{o}$

Where $c_{0}$ is the damping coefficient and $f_{c}$ is the frictional force, which is related to the fluid yield stress. An offset in the force $f_{0}$ is included to account for the nonzero mean observed in the measured force due to the presence of the accumulator. Recalling that at the beginning and ending of piston stroke where the velocity of the piston is zero, the force generated in the frictional element is equal to the applied force.

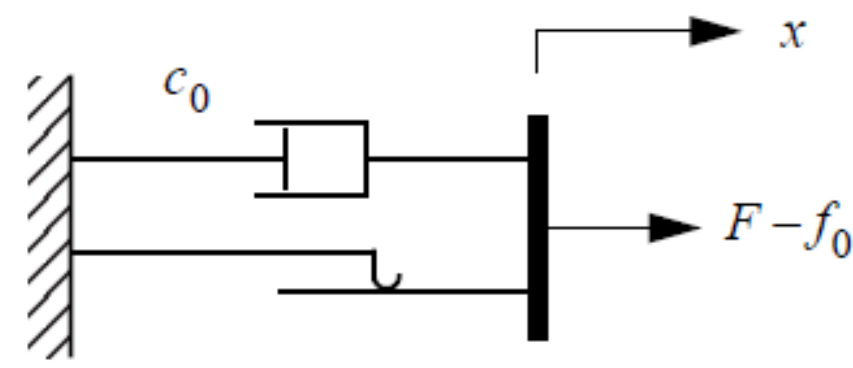

Fig. 7. Bingham model of a controllable fluid damper (Stanway, et al. 1985, 1987).

The SIMULINK program in MATLAB is used to simulate the performance of the MR damper described by Bingham model. Figure (8) shows the block diagram of the SIMULINK model used to describe the MR damper performance. 


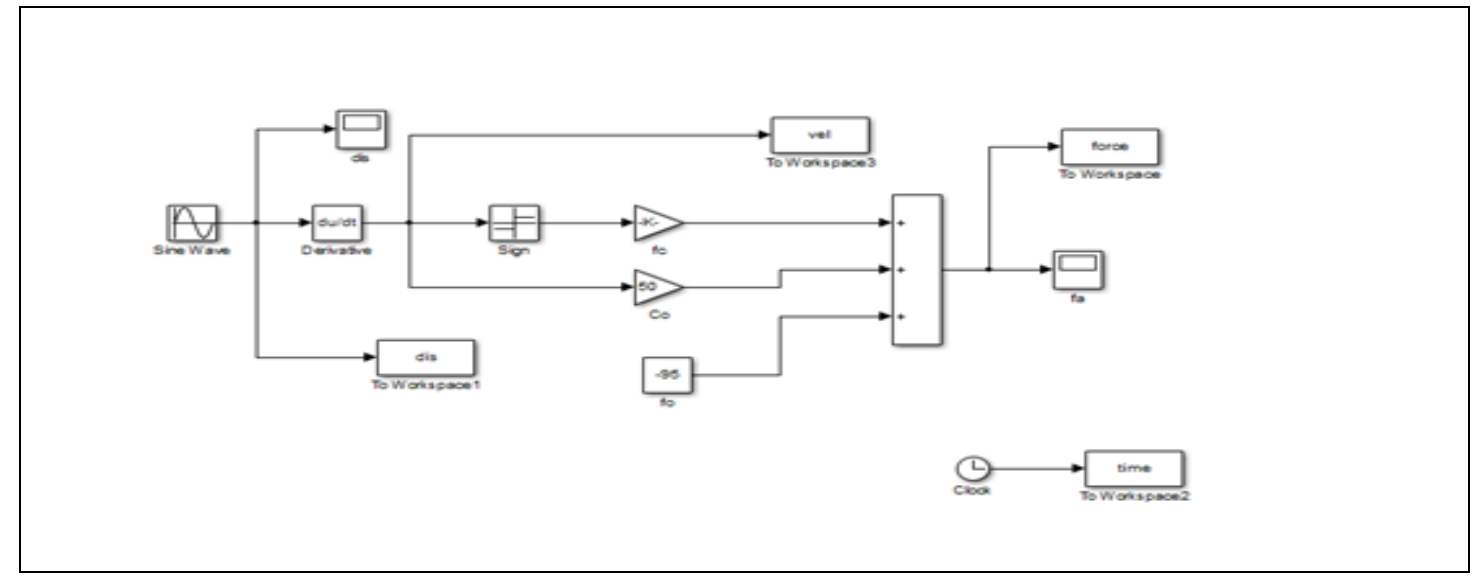

Fig. 8. block diagram of SIMULINK Bingham model.

The model was fitted to $2 \mathrm{~Hz}$ sinusoidal response data and the command voltage to the current driver was a constant $3.5 \mathrm{~V}$. The parameter described in equation (1) are chosen from [11, $12], f_{c}=670 \mathrm{~N}, c_{0}=50 \mathrm{~N} \mathrm{sec} / \mathrm{cm}$ and $f_{0}=-95 \mathrm{~N}$. Figure (9) shows a comparison between the predicted and experimentally obtained responses. Although the force-displacement behavior appears to be reasonably modeled, examination of the force-velocity response and the temporal variation of the force show that the behavior of the damper is not captured, especially for velocities that are near zero. In particular, this model does not exhibit the nonlinear force-velocity response observed in the data for the case when the acceleration and velocity have opposite signs (or alternatively, when the velocity and the displacement have the same sign) and when the magnitudes of the velocities are small. While this model may be convenient for response analysis, it is not suitable for control analysis. Furthermore, at zero velocity, the measured force has a positive value when the acceleration is negative (positive displacements), and a negative value when the acceleration is positive (negative displacement). This behavior must be captured in a mathematical model to adequately characterize the device for control applications.

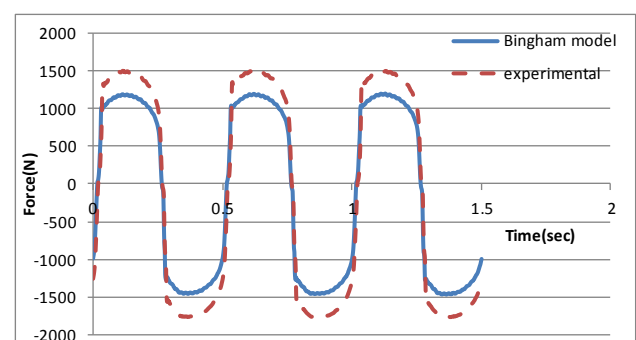

Force Vs time

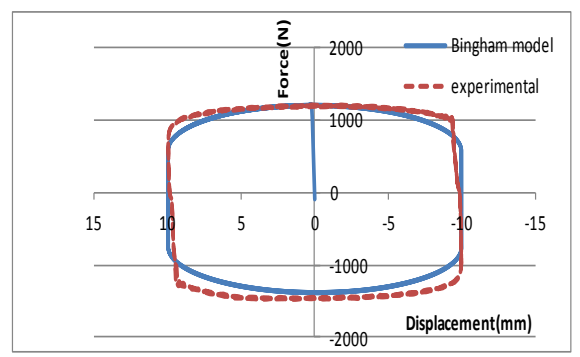

Force Vs displacement

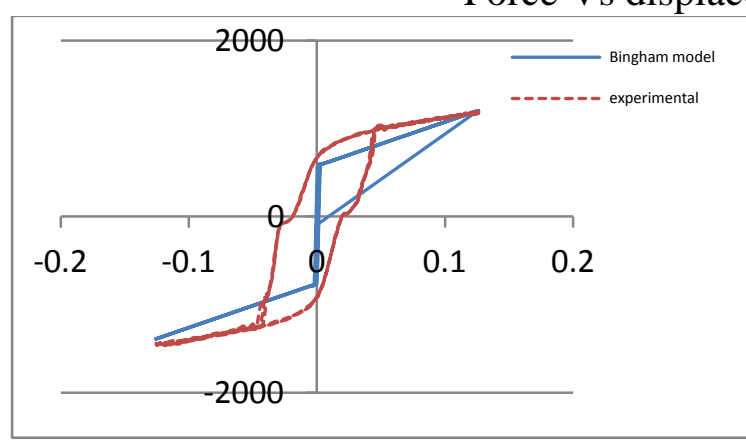

Force Vs velocity

Fig.0. Comparison between the predicted and experimentally obtained responses for the Bingham model. 
Gamota and Filisko (1991) proposed an extension of the Bingham model, which is given by the viscoelastic-plastic model shown in figure (10). The model consists of the Bingham model (i.e., a frictional element in parallel with a dashpot) in series with a standard model of a linear solid (Shames and Cozzarelli, 1992).

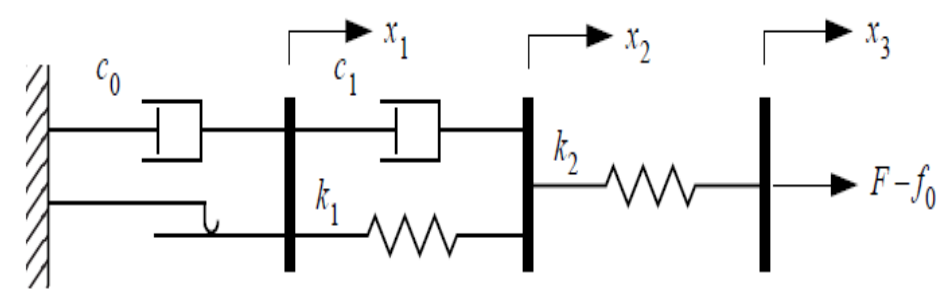

Fig. 10. Model proposed by Gamota and Filisko (1991).

The governing equations for this model are given by:

$$
\begin{aligned}
& \left.\begin{array}{c}
F=k_{1}\left(x_{2}-x_{1}\right)+c_{1}\left(\dot{x}_{2}-\dot{x}_{1}\right)+f_{o} \\
=c_{o} \dot{x}_{1}+f_{c} \operatorname{sgn}\left(\dot{x}_{1}\right)+f_{o} \\
=k_{2}\left(x_{3}-x_{2}\right)+f_{o}
\end{array}\right\}, \quad|F|>f_{c} \\
& \left.\begin{array}{c}
F=k_{1}\left(x_{2}-x_{1}\right)+c_{1}\left(\dot{x}_{2}\right)+f_{o} \\
=k_{2}\left(x_{3}-x_{2}\right)+f_{o}
\end{array}\right\}, \quad|F| \leq f_{c}
\end{aligned}
$$

Where $c_{0}$ is the damping coefficient associated with the Bingham model and $k_{1}, k_{2}$, and $c_{1}$ are associated with the linear solid material. Note that when $|F| \leq f_{c}, \dot{x}_{1}=0$.

The parameters chosen are $[11,12] f_{c}=670 \mathrm{~N}, c_{0}=5000 \mathrm{~N} \mathrm{sec} / \mathrm{cm}, c_{1}=1300 \mathrm{~N} \mathrm{sec} / \mathrm{cm}$, $k_{1}=5 \times 10^{4} \mathrm{~N} / \mathrm{cm}, k_{2}=2 \times 10^{6} \mathrm{~N} / \mathrm{cm}$ and $f_{0}=-95 \mathrm{~N}$. A comparison between the predicted responses and the corresponding experimental data is provided in figure (11).
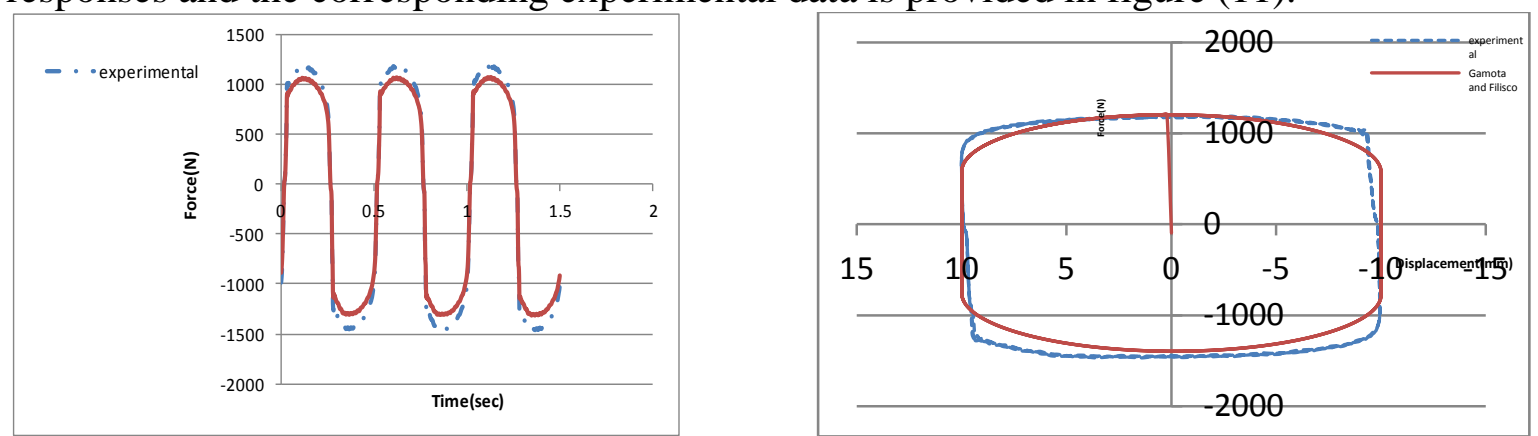

Force Vs Time

Force Vs displacement

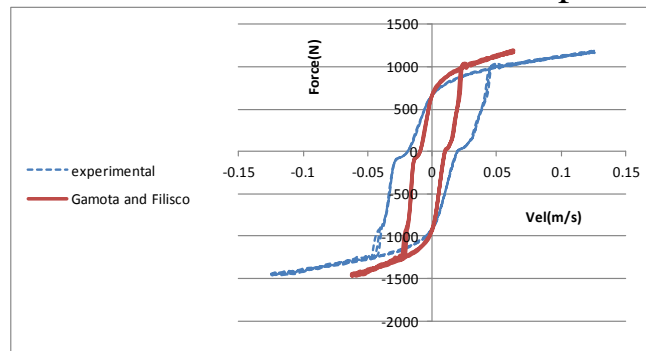

Force Vs velocity

Fig. 11. Comparison between the predicted and experimentally obtained responses for the Gamota and Filisko Model. 
This model can portray the force-displacement behavior of the damper well. In addition, it possesses force-velocity behavior that more closely resembles the experimental data.

One model that is numerically tractable and has been used extensively for modeling hysteretic systems is the Bouc-Wen model (Wen 1976). The Bouc-Wen model is extremely versatile and can exhibit a wide variety of hysteretic behavior. A schematic of this model is shown in figure (12). The force in this system is given by

$F=c_{o} \dot{x}+k_{o}\left(x-x_{o}\right)+\alpha z$

Where the evolutionary variable $\mathrm{z}$ is governed by:

$\dot{z}=-\gamma|\dot{x}||z| z^{n-1}|-\beta \dot{x}| z^{n} \mid+A \dot{x}$

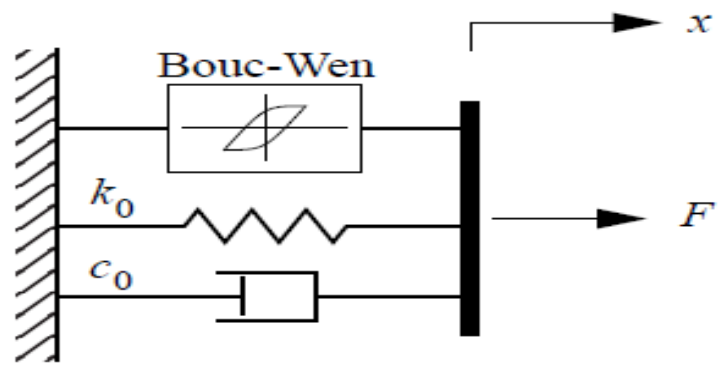

Fig. 12. Bouc-Wen model of the MR damper.

By adjusting the parameters of the model $\gamma, \beta$ and $\mathrm{A}$, one can control the linearity in the unloading and the smoothness of the transition from the pre-yield to the post-yield region. In addition, the force $f_{0}$ due to the accumulator can be directly incorporated into this model as an initial deflection $x_{0}$ of the linear spring $k_{0}$.

A set of parameters was determined to fit the response of the Bouc-Wen model to the experimentally measured response of the MR damper. The parameters for the model were chosen from $[11,12], \alpha=880 \mathrm{~N} / \mathrm{cm}, c_{0}=50 \mathrm{~N} \mathrm{sec} / \mathrm{cm}, k_{0}=25 \mathrm{~N} / \mathrm{cm}, \gamma=100 \mathrm{~cm}^{-2}, \beta=100 \mathrm{~cm}^{-}$ ${ }^{2}, n=2, A=120$, and $x_{0}=3.8 \mathrm{~cm}$. A comparison between the predicted responses and the corresponding experimental data is provided in figure (13), The Bouc-Wen model predicts the force-displacement behavior of the damper well, and it possesses force-velocity behavior that more closely resembles the experimental data. However, similar to the Bingham model, the nonlinear force-velocity response of the Bouc-Wen model does not roll-off in the region where the acceleration and velocity have opposite signs and the magnitude of the velocities are small.

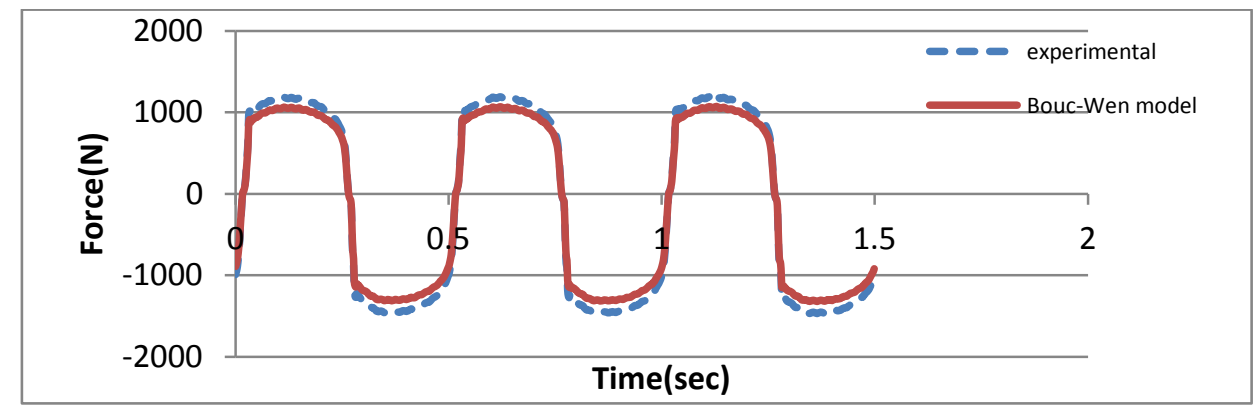

Force Vs time 


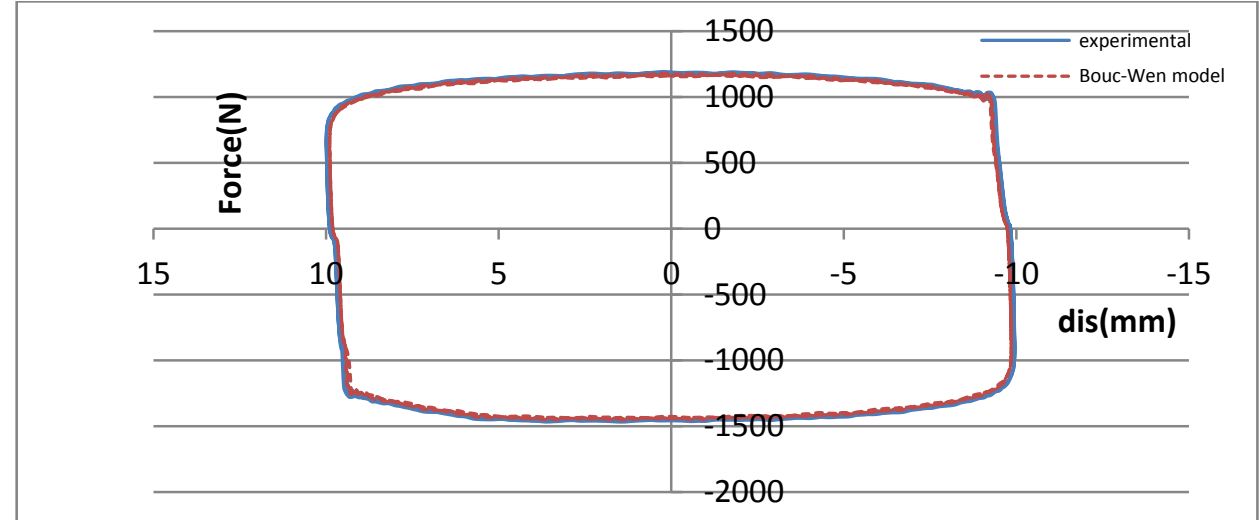

Force Vs displacement

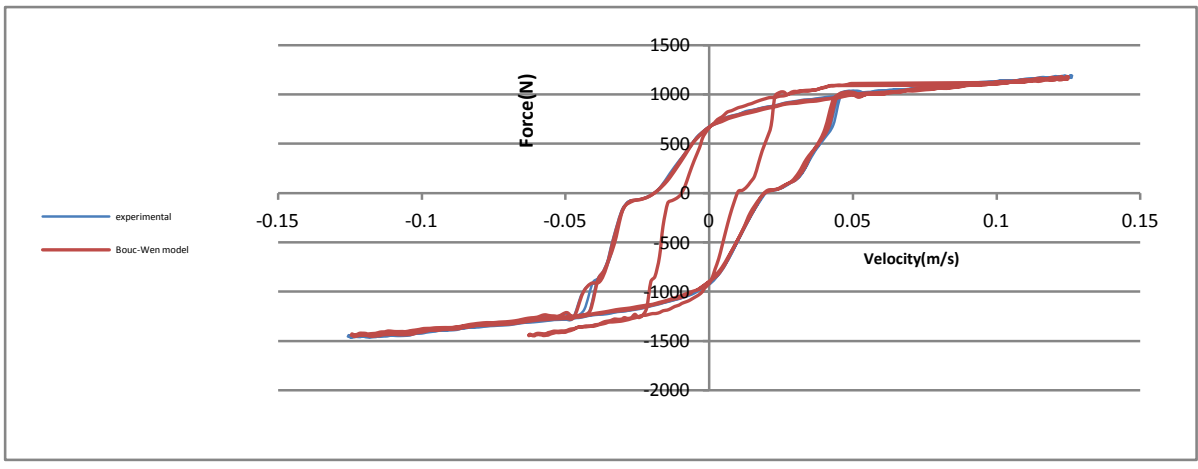

Force Vs velocity

Fig. 13. Comparison between the predicted and experimentally obtained responses for the Bouc-Wen Model.

To better predict the damper response in this region, a modified version of the system is proposed, as shown in figure (14). To obtain the governing equations for this model, consider only the upper section of the model. The forces on either side of the rigid bar are equivalent; therefore,

$c_{1} \dot{y}=\alpha z+k_{o}(x-y)+c_{o}(\dot{x}-\dot{y})$

Where the evolutionary variable $z$ is governed by

$\dot{z}=-\gamma|\dot{x}-\dot{y}||z| z^{n-1} \mid-\beta\left(\dot{x}-\dot{y}\left|z^{n}\right|+A(\dot{x}-\dot{y})\right.$

Solving for $y^{\cdot}$ results in

$\dot{y}=\frac{1}{\left(c_{o}+c_{1}\right)}\left\{\alpha z+c_{o} x+k_{o}(x-y)\right\}$

The total force generated by the system is then found by summing the forces in the upper and lower sections of the system in figure (14) yielding:

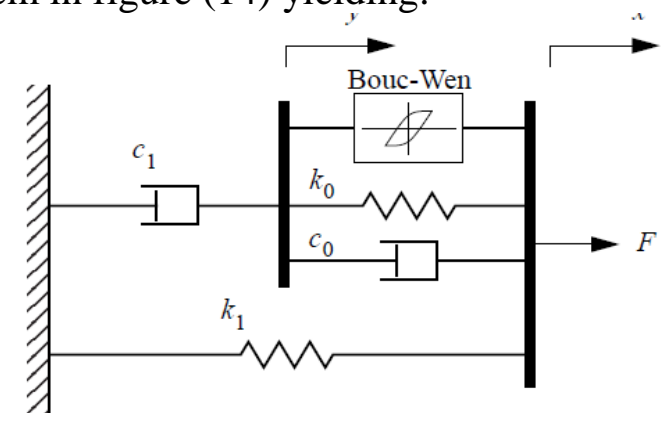

Fig.14 . modified Bouc-Wen MR damper model. 
$F=\alpha z+c_{o}(\dot{x}-\dot{y})+k_{o}(x-y)+k_{1}\left(x-x_{o}\right)$

The total force can also be written as

$F=c_{1} \dot{y}+k_{1}\left(x-x_{o}\right)$

In this model, the accumulator stiffness is represented by $k_{1}$ and the viscous damping observed at larger velocities is represented by $c_{0}$. A dashpot, represented by $c_{1}$, is included in the model to produce the roll-off that was observed in the experimental data at low velocities $k_{0}$, is present to control the stiffness at large velocities, and $x_{0}$ is the initial displacement of spring $k_{1}$ associated with the nominal damper force due to the accumulator.

The parameters for this model were chosen to be $\alpha=963 \mathrm{~N} / \mathrm{cm}, c_{0}=53 \mathrm{~N} \mathrm{sec} / \mathrm{cm}, k_{0}=$ $14 \mathrm{~N} / \mathrm{cm}, c_{1}=930 \mathrm{~N} \mathrm{sec} / \mathrm{cm}, k_{1}=5.4 \mathrm{~N} / \mathrm{cm}, \gamma=200 \mathrm{~cm}^{-2}, \beta=200 \mathrm{~cm}^{-2}, n=2, A=207$, and $x_{0}=$ $18.9 \mathrm{~cm}$, which fit the response of the proposed model. A comparison between the predicted responses and the corresponding experimental data is provided in figure (14). The proposed model for the damper predicts the behavior of the damper very well in all regions, including in the region where the acceleration and velocity have opposite signs and the magnitude of the velocities are small.
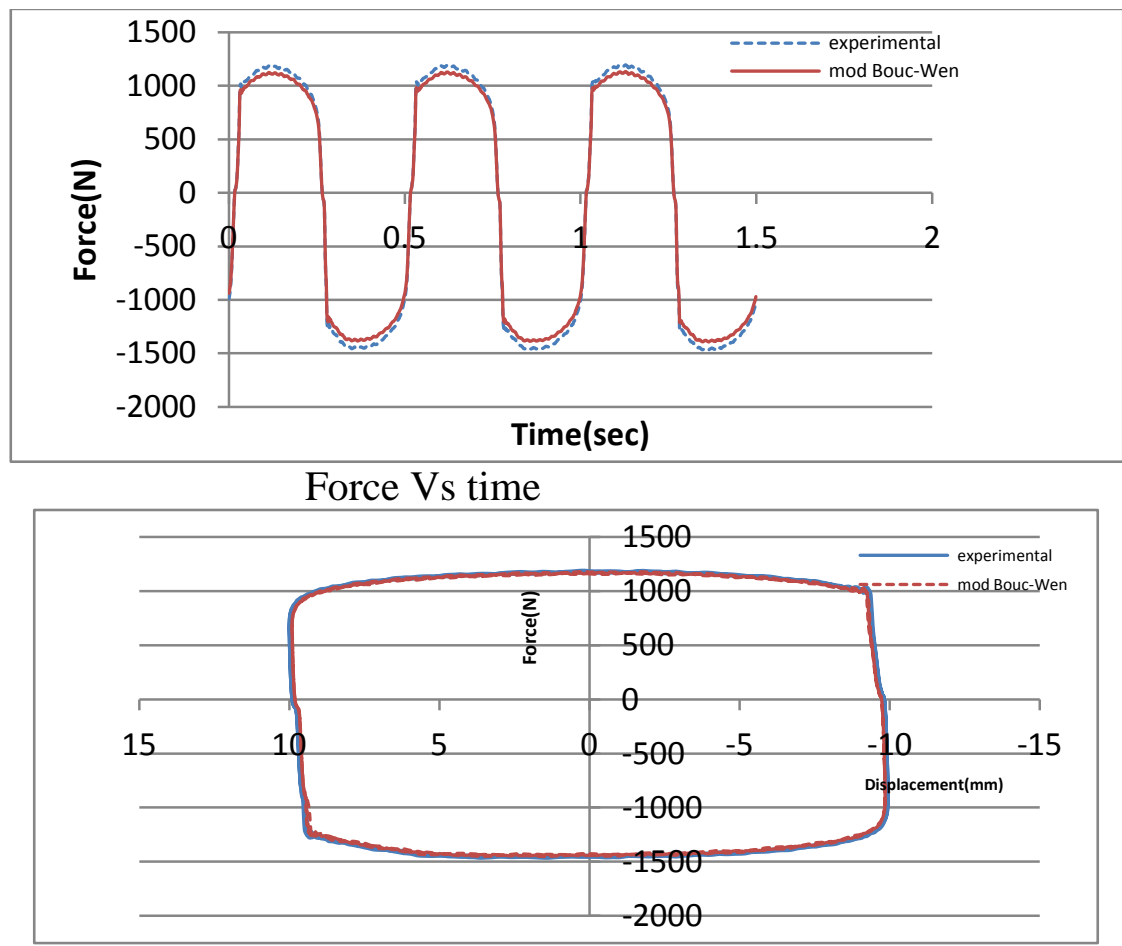

Force Vs displacement

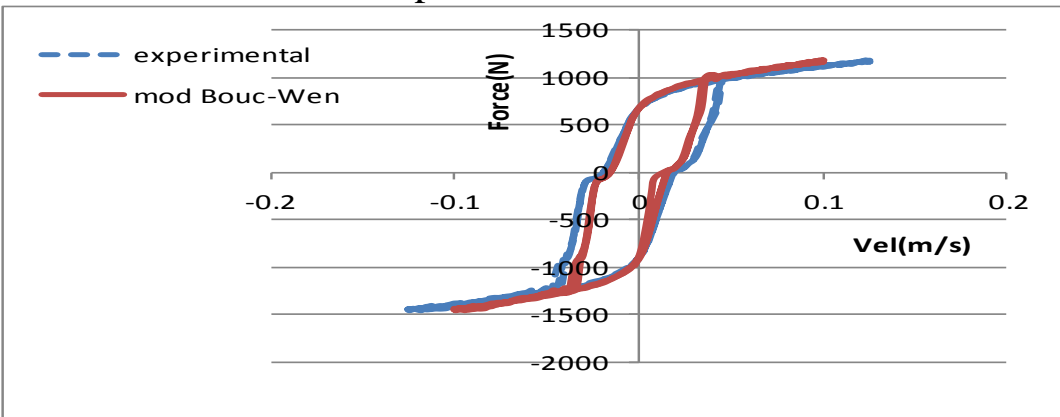

Force Vs velocity

Fig. 15. Comparison between the predicted and experimentally obtained responses for the proposed model. 


\section{Conclusion}

A review of several idealized mechanical models for controllable fluid dampers has been presented.

Subsequently, one of these models has been proposed that overcomes a number of the shortcomings of these models and can effectively portray the behavior of a typical magnetorheological damper especially in dynamic characteristics. This model is based on a modified Bouc-Wen hysteresis model, which is numerically tractable and is capable of exhibiting a wide variety of hysteretic behaviors. A dashpot has been added in series with the Bouc-Wen model which creates the nonlinear roll-off observed in the force as the velocity approaches zero; and an additional spring is incorporated into the model to account for the stiffness of the accumulator present in the prototype MR damper. When compared with experimental data, the modified Bouc-Wen model was shown to accurately predict the response of the MR damper over a wide range of operating conditions. These results indicate that the model can be effectively used for control algorithm development and system evaluation.

\section{References}

1- Winslow, W. M. Method and means for translating electrical impulses into mechanical force 1947, U.S. Patent 2,417,850.

2- Winslow, W. M. Induced fibration of suspensions 1949, Journal of applied physics, Volume: 20, pp 1137-1140.

3- Weiss, K. D. \& Nixon, D. A. Viscoelastic properties of magneto- and electrorheological fluids 1994, Journal of Intelligent Material Systems and Structures, Volume: 5, pp 772775 .

4- Fernando, D., Goncalves, J. H. K., \& Mehdi, A. A review of the state of the art in magnetorheological fluid technologies - Part I: MR fluid and MR fluid models 2006, the Shock and Vibration Digest, Volume: 38, (3), pp 203-219.

5- Wang, J. \& Meng, G. Magnetorheological fluid devices: principles, characteristics and applications in mechanical engineering 2001, Proceedings of the Institution of Mechanical Engineers; Part B; Journal of Engineering Manufacture Volume: 215 (3) pp 165- 174.

6-Bossis, G. and Lemaire, E. (1991). "Yield Stresses in Magnetic Suspensions," Journal of Rheology, Vol 35(7), pp. 1345-1354.

7-Carlson, J.D. (1994). "The Promise of Controllable Fluids." Proc. of Actuator 94 (H. Borgmann and K. Lenz, Eds.), AXON Technologie Consult GmbH, pp. 266-270.

8-Carlson, J.D. and Weiss, K.D. (1994). "A Growing Attraction to Magnetic Fluids," Machine Design, August, pp. 61-64.

9-Carlson, J.D., Catanzarite, D.M. and St. Clair, K.A. (1995). "Commercial MagnetoRheological Fluid Devices," Proceedings of the 5th International Conference on ER Fluids, MR Fluids and Associated Technology, U. Sheffield, UK.

10- Constantinou, M.C. and Symans, M.D., (1994). "Semi-Active Fluid Viscous Dampers for Seismic Response Control." Proc. of the First World Conference on Structural Control, Pasadena, CA.

11- Grasselli, Y., Bossis, G. and Lemaire, E. (1993). "Field-Induced Structure in Magnetorheological Suspensions." Progress in Colloid \& Polymer Science, Vol. 93, p. 175.

12- Kabakov, A.M. and Pabat, A.I. (1990). "Development and Investigation of Control Systems of Magnetorheological Dampers." Soviet Electrical Engineering, Vol. 61, No. 4, p. 55. 